\title{
Year Zero o cómo Nine Inch Nails rompió las barreras de Europa con una experiencia narrativa ${ }^{1}$
}

\section{Hernán David Espinosa-Medina² Ana María Pérez-Guerrero ${ }^{3}$}

Recibido: 2016-01-21

Enviado a pares: 2016-01-21
Aprobado por pares: 2016-03-08

Aceptado: 2016-04-03

DOI: 10.5294/pacla.2017.20.2.4

Para citar este artículo / to reference this article / para citar este artigo

Espinosa-Medina, H. D. y Pérez Guerrero, A. M. (2017). Year Zero o cómo Nine Inch Nails rompió las barreras de Europa con una experiencia narrativa. Palabra Clave, 20(2), 360388. DOI: 10.5294/pacla.2017.20.2.4

\section{Resumen}

El presente artículo tiene por objeto el estudio de las estrategias empleadas en el proceso creativo de la experiencia del alternate reality game (ARG) del álbum Year Zero (2007), de la agrupación Nine Inch Nail, como un caso de estudio de los aspectos por tener en cuenta a la hora de introducir un producto en el mercado global. Para ello, se realiza un marco teórico de los procesos de transducción cultural y, en particular, se expone el modelo analítico de los elementos esenciales en los contenidos transparentes basado en el mitotipo. Finalmente, se realiza una exploración de la morfología del

1 Este artículo es producto de la investigación Cultural Transductions Universals and Lacunae: An International Contra-flow Study (Código COM 62-2013) adscrito al grupo de investigación Cultura Audiovisual de la Facultad de Comunicación de la Universidad de La Sabana, financiado por el Fondo Patrimonial para la Investigación de la Universidad de La Sabana.

2 Universidad de La Sabana, Colombia. hernanem@unisabana.edu.co

3 Universidad de La Sabana, Colombia. ana.perez@unisabana.edu.co 
mitotipo en las acciones que compusieron la experiencia ya mencionada, para concluir con la importancia de apelar a una estrategia que incluya la calidad de los valores de producción y, en especial, en apelar al componente antropológico de narrativas clásicas, asentadas en la realidad.

\section{Palabras clave}

Transmedia; transducción cultural; transparencia cultural; cultural shareability; universales y lagunas culturales (Fuente: Tesauro de la Unesco). 


\section{Year Zero or How Nine Inch Nails Broke the Barriers of Europe with a Narrative Experience}

\section{Abstract}

The present article aims to study the strategies used in the creative process of the experience of the alternate reality game (ARG) of the album Year Zero (2007), by the band Nine Inch Nails, as a case study of the aspects to take into account when introducing a product in the global market. For this purpose, a theoretical framework of cultural transduction processes is carried out and, in particular, the analytical model of the essential elements in the transparent contents based on the mythotype is presented. Finally, an exploration of the morphology of the mythotype in the actions that composed the already mentioned experience is carried out, in order to conclude with the importance of appealing to a strategy that includes the quality of the values of production and, in particular, to the anthropological component of classic narratives, based on reality.

\section{Keywords}

Transmedia; cultural transduction; cultural transparency; cultural shareability; universals and cultural gaps (Source: Unesco Thesaurus). 


\section{Year Zero ou como Nine Inch Nails rompeu as barreiras na Europa com uma experiência narrativa}

\section{Resumo}

O presente artigo tem como objeto o estudo das estratégias empregadas no processo criativo da experiência do alternate reality game (ARG) do álbum Year Zero (2007), da banda Nine Inch Nail, como um caso de estudo dos aspectos por levar em consideração na hora de introduzir um produto no mercado global. Para isso, se realiza um marco teórico dos processos de transdução cultural e, em particular, se expõe o modelo analítico dos elementos essenciais nos conteúdos transparentes baseado no mitotipo. Finalmente, se realiza uma exploração da morfologia do mitotipo nas ações que compuseram a experiência já mencionada, para concluir com a importância de apelar para uma estratégia que inclua a qualidade dos valores de produção e, especialmente, em apelar o componente antropológico de narrativas clássicas, assentadas na realidade.

\section{Palavras-chave}

Transmídia; transdução cultural; transparência cultural; cultural shareability; universais e lacunas culturais (Fonte: Tesauro da Unesco). 
En el mundo del entretenimiento contemporáneo, es entendible que una de las metas de negocio de muchos productores sea la internacionalización del contenido que desarrollan. Desde el estudio de Hollywood más grande y las disqueras más poderosas, hasta los artistas independientes, la posibilidad de trascender ciertas fronteras y alcanzar nuevos mercados es una marca importante por superar. Poder abrirse a los mercados internacionales representa, sin lugar a dudas, la posibilidad de explotar fuentes económicas nuevas y amplias, pero, además, en el caso de muchos artistas, también representa la promesa de la globalización, de que su mensaje y su voz serán escuchados por tantas personas como sea posible.

Ese mundo globalizado es el contexto en el que Wright (2011) ubica el lanzamiento, en 2007, del álbum musical Year Zero, producido por Trent Reznor, miembro principal de la agrupación Nine Inch Nails. Para Wright, es importante destacar la forma en que este disco se enmarca en la era de la convergencia de medios descrita por Jenkins (2006a) y también en medio de un contexto histórico particular: el difícil momento que se vivía, por las medidas de control que el Gobierno de los Estados Unidos adoptó después de los acontecimientos del 9-11. Asimismo, este es el mundo en que las disqueras y otros actores dominantes en la industria del entretenimiento intentaban implementar políticas de intervención, que les permitieran contrarrestar los efectos no deseables para su negocio, que producían las dinámicas que los usuarios de aplicaciones, como Napster, estaban generando, gracias a las posibilidades que surgían con internet.

En su momento, Reznor decidió buscar la manera de oponerse ideológicamente a los esquemas de distribución tradicionales de la industria musical, hasta el punto de que durante un concierto en Sídney, en 2007, instó a sus fanes a copiar y compartir ilegalmente su álbum (Brown, 2011, p. 199). Esta resistencia y el deseo de dar a su audiencia más de su trabajo llevaron al artista a buscar una manera diferente de hacer llegar su música y sus ideas en el ámbito global, experimentando con las posibilidades que ofrecían las nuevas tecnologías. Así es como el músico, junto con la agencia 42 Entertainment, crea una experiencia virtual para involucrar a sus fanes en el lanzamiento de Year Zero, cuyo éxito internacional constituye un 
caso paradigmático de la promoción discográfica y de la creación de los juegos de realidad aumentada (ARG 'augmented reality games'), así como de las narrativas transmedia.

Aunque el fenómeno que significó esta experiencia ha despertado el interés de algunos autores, como Wright (2011) o Brown (2011), lo cierto es que aún faltan estudios que aborden su narrativa respecto de la transducción cultural (Uribe-Jongbloed y Espinosa-Medina, 2014). Por eso, aquí nos interesa analizar las estrategias empleadas en su creación, enfoncándonos de manera especial en cómo estas permitieron a Reznor llegar a una audiencia compuesta por gentes de diferentes geografías y contextos culturales, a través de la distribución independiente.

Para ello, en primer lugar, se hace una revisión de algunos de los conceptos que se han desarrollado desde la academia, en el ámbito del estudio de los flujos y contraflujos internacionales de las industrias del cine y la televisión, para dar una visión global de en qué consiste la transducción cultural, y así abordar los elementos que permitieron la comercialización y distribución exitosa del álbum en el mercado internacional. En segundo lugar, en el marco conceptual de la transducción cultural nos enfocamos en el concepto de transparencia, acuñado por Olson (1999), con el fin de presentar los elementos textuales que operan en la compartibilidad de los contenidos. A continuación, se expone el modelo de análisis adoptado para el estudio de Year Zero, a partir de la morfología del mitotipo, para finalmente aplicarlo en el caso de estudio.

\section{Ideas sobre la transducción cultural}

La transducción cultural, o la acción que supone introducir un producto de entretenimiento en un mercado cultural, ha sido designado con diferentes nombres y ha sido abordado desde diversas perspectivas, como un proceso de adaptación de una obra de un contexto particular a otro (Bartrina, 2004; Cattrysse, 1992; Chiaro, 2008; Conway, 2012; Hogg, 2013; Sakellariou, 2014). Gracias a esta multiplicidad de visiones, al intentar articularlas bajo un solo corpus teórico, se distinguen varios elementos por tener en cuenta sobre el por qué, quiénes se involucran en el proceso y, en particular, sobre el 
cómo se da el proceso de transducción (Uribe-Jongbloed y Espinosa-Medina, 2014). En este caso, nos interesa ahondar en algunos de los aspectos que tienen que ver con el cómo de este proceso.

Ahora bien, tradicionalmente se ha visto cómo ciertos contenidos son desarrollados por productores locales, con un posicionamiento particular en un mercado nacional o regional, y que luego, dependiendo de su éxito, se trata de vender a otros mercados para ampliar su margen de rentabilidad (Davis y Nadler, 2010; Lee, 2006; Rivera-Betancur y Uribe-Jongbloed, 2012). Esto ha llevado a que los productores busquen crear productos que pretenden alcanzar mercados internacionales desde su concepción o a que adopten y evolucionen dichas estrategias de internacionalización de los productos en el ecosistema mediático contemporáneo.

Visto desde este prisma, y analizando múltiples casos de las industrias del cine, la televisión y las publicaciones de prensa, se ve cómo algunos autores han establecido, entre varios factores, ciertas estrategias para facilitar la inserción de productos de entretenimiento en mercados culturales. Dichas estrategias van más allá de identificar las posibles ocasiones en que la transducción cultural se daría con menores riesgos (Fung, 2004; Lee, 2006; Straubhaar, 1991) y procuran establecer bases para la transformación del producto como tal para cercarlo a la audiencia objetivo. Buscando entender cómo funcionan estas estrategias, tendremos en cuenta para el análisis presentado más adelante las definiciones de lagunas y universales (Rohn, 2011), compartibilidad cultural o shareability (Singhal y Svenkerud, 1994; Singhal y Udornpim, 1997), así como la noción de transparencia cultural (Olson, 1999), y cómo se relacionan entre sí en la transducción cultural. Una vez definidos estos conceptos, se espera evidenciar cómo se emplean estas estrategias en un caso particular, que, además, permite ampliar su significado gracias a su reapropiación en el contexto mediático contemporáneo por parte del productor de contenido.

\section{Universales y lagunas culturales}

Los conceptos de lagunas y universales culturales, planteados y desarrollados por Ulrike Rohn (2011), aluden a las características de un texto que pue- 
den alejarlo o acercarlo a una determinada audiencia o mercado. Cabe notar que estos rasgos son solo del contenido y no necesariamente del mercado en el que se originaron. Así, estas nociones permiten el análisis de productos que, desde su concepción, se proyectan para alcanzar contextos diferentes del de sus creadores. Por otro lado, también es importante recalcar que esta perspectiva favorece el estudio de casos, en el que el concepto del producto trasciende la obra y abarca otros elementos textuales, que se desarrollan y giran en torno a esta, y que le ayudan a dar significado, como los elementos paratextuales creados por los productores y los consumidores (Gray, 2010, p. 6). Esto es importante, ya que el análisis contempla entre sus categorías estas otras piezas textuales y resalta su importancia en la adopción del texto por parte de la audiencia.

Dicho lo anterior, podemos expandir estas categorías de análisis. Por un lado, se encuentran los universales culturales, que son aquellas características del texto que lo acercan a una audiencia particular. Dentro de ellas, se encuentran tres categorías: universales de contenido, universales generados por la compañía y universales creados por el consumidor. La primera de estas, los universales del contenido, hace referencia a aquellos aspectos del producto como tal que son entendibles e interpretables por la audiencia dado su capital cultural (Bourdieu, 1984), y que también se puede abordar desde la noción de intertextualidad como elemento básico para dar significado a un contenido en un contexto particular (Allen, 2011).

Los universales generados por la compañía son aquellos elementos paratextuales, que los productores y distribuidores generan para acercar su producción a la audiencia, ya sea suscitando una expectativa, con miras a lograr una proximidad deseada (Iwabuchi, 2010), ya sea una expansión del producto en el contexto de la audiencia, en los dos frentes descritos por Marc Steinberg (2012, p. 167): de manera extensiva generando un número cada vez mayor de consumidores en un mercado o intensiva aumentando el grado del consumo de productos paratextuales o del contenido en la vida del público.

Por último, los universales generados por el consumidor representan las apropiaciones que nacen de la propia audiencia y que van desde simples 
traducciones hasta paratextos producidos por las comunidades de usuarios. De este modo, se favorece el análisis y la atención sobre los elementos que acentúan lo que Jenkins, Ford y Green (2013) denominan la esparcibilidad o spredability.

En contraste con estas primeras características, se encuentran las lagunas culturales, que son aquellas características que alejan el contenido de la audiencia. En este caso, Rohn también identifica tres categorías. En primer lugar, están las lagunas de contenido, que son elementos directos del texto que resultan irrelevantes para un mercado particular o que pueden llegar inclusive a generar rechazo, como el uso de un concepto moral que se considere inapropiado o un personaje que no exista ni tenga ninguna conexión con un entorno determinado. Por otra parte, están las lagunas de producción, que tienen que ver con la materialidad del contenido. Es decir, con el uso de elementos, como el formato, la iluminación, el arte, el sonido, los planos y demás componentes estéticos que pueden ser percibidos como distintos, de mala calidad según estándares disímiles o simplemente extraños. Por último, se encuentran las lagunas de capital, que aparecen cuando la audiencia en un mercado puede tener interés en una producción, la puede encontrar estéticamente atrayente, pero carece del capital cultural requerido para decodificarlo. Un ejemplo de esto puede ser la falta del manejo del idioma o la falta de ciertos referentes.

Cuando se intenta insertar un producto de entretenimiento en distintos mercados culturales, hay que tener claros los universales y las lagunas de ese contenido, en relación con esos mercados, para abordar el proceso de implantación, intentando mantener los universales que ya existen y tratando de cambiar o eliminar las lagunas que se identifiquen. Este proceso ha sido descrito respecto de deculturización, eliminación de elementos culturales particularmente evidentes en un producto, que puedan marcar una distancia con las audiencias en los mercados que se busca alcanzar, para lograr un producto aculturizado, que carece de elementos que pudieran ser leídos como foráneos o ajenos por la audiencia en un mercado particular, y que finalmente es reculturizado, es decir que es adaptado para apelar de manera más fuerte y directa a la nueva audiencia (Wang y Yeh, 2005). 
Entendemos, pues, que estos son elementos particulares del texto que apuntan a acercarlo o a erradicar distancias entre este y el mercado objetivo. Sin embargo, no todos los mercados son iguales y no todos los productores tienen las mismas capacidades. Esto ha derivado en el surgimiento de, al menos, dos aproximaciones al proceso de transducción respecto de lo que podríamos llamar estrategias de transducción. Por un lado, hay quienes optan por la compartibilidad cultural y, por el otro, quienes abordan el proceso desde la transparencia cultural, entendiendo que en ningún momento se plantean estas dos estrategias como categorías discretas, ni excluyentes.

\section{Compartibilidad (shareability), aproximación desde las particularidades del concepto}

La compartibilidad suele darse en los casos en los que los productores no tienen gran capacidad de distribución internacional o cuando se estima que un determinado producto puede ser atractivo para algunas audiencias particulares por razones características y no para las audiencias de regiones geolingüísticas o culturales extensas. En estos casos, quien tiene derechos sobre la propiedad tiende a buscar socios en cada uno de los mercados particulares en los que quiere insertar el producto. El interés de la búsqueda puede estar basado, en parte, en el interés de que dichos socios asuman parte del riesgo de la inversión, pero también se da en casos en que este tipo de asociaciones permite sobrepasar ciertas barreras legales en países en los que existen medidas que buscan la protección de la industria nacional. Al mismo tiempo, se espera que los productores locales traigan a la mesa sus conocimientos sobre cada uno de los mercados particulares para fortalecer el proceso de reculturización en cada caso particular y, así, mitigar el riesgo de inversión (Davis y Nadler, 2010). En estos casos, el desarrollo de elementos, como los formatos, ha sido un gran avance, que ha permitido abrir nuevas fuentes de rentabilidad para productores que antes no tenían fácil acceso a mercados internacionales (Moran, 2009; Straubhaar, 2007).

En la transducción cultural, esta estrategia en la que se pretende acercar cada uno de los mercados culturales, aumentando los universales con cada contexto en particular, es lo que se denomina compartibilidad cultural 
o cultural shareability (Singhal y Svenkerud, 1994; Singhal y Udornpim, 1997; Uribe-Jongbloed y Espinosa-Medina, 2014).

Por otro lado, un producto puede presentar aspectos que lo hacen atractivo a un gran número de mercados a la vez. Esto no quiere decir necesariamente que todos los elementos lo hagan atrayente a todos los mercados de la misma manera o que el producto sea culturalmente neutro, sino que sus componentes permiten la apropiación del texto por parte de múltiples audiencias en simultáneo.

\section{Mitotipo, elemento clave de la transparencia cultural}

El concepto de transparencia cultural, propuesto por Scott Robert Olson en Hollywood Planet (1999), alude a la doble estrategia, propia de los contenidos culturales de los mercados núcleo (Uribe-Jongbloed y EspinosaMedina, 2014, p. 26), en concreto, del estadounidense, que consiste en la combinación de los aspectos particulares de su cultura, para garantizar una conexión estrecha entre sus producciones y la audiencia local, y el uso del mitotipo como elemento universal que le permite alcanzar un público internacional.

Es en esta última táctica, en la que se centrará nuestra atención, al ser la canalizadora de transparencia, la cual permite abordar el estudio de universales de contenido, de universales generados por la compañía y de universales creados por los consumidores, a través de un modelo analítico, que se desarrolla en el siguiente apartado, y que aborda la morfología de los textos transparentes.

Si bien los mitos "son los modelos universales de la experiencia humana” (Place, 1974, p. 4), para Olson representan también la resolución de algunas de las necesidades del hombre propuestas por Maslow (1987): básicas, de seguridad y protección, sociales o de filiación y de estima o reconocimiento. Sin embargo, como el mito está dotado de una iconografía y de rasgos propios de la cultura en la que surge como tal, los contenidos basados en ellos necesitan, para su universalización, la presencia del mitotipo. 
Este concepto, tomado de Work on the Myth, de Hans Blumenberg (1985, p. 4), se refiere al sistema de expectativas afectivas y de cumplimientos que subyacen en la función del mito, de distanciar al hombre del absolutismo de la realidad; es decir, de la idea de que toda persona tiene respecto de su falta de control sobre las condiciones de su propia existencia. Por tanto, lo que el mitotipo viene a proporcionar es una impresión de orden o de sentido de la vida o, en palabras de Roncallo-Dow (2008):

\begin{abstract}
La "apropiación antropomórfica" del mundo sugiere ante todo un trabajo de continua y constante traducción en la que lo desconocidoaterrador sea descifrado, recodificado y susceptible de ser decantado y asimilado; el trabajo de traducción es una respuesta al terror original aprehensible desde la relación cotidiana con la ferocidad del propio entorno y se centra en la interacción de la audiencia con el texto. [...] La toma de distancia tiene que ver, entonces, con una fundamental necesidad de resguardo, de conservación, con una especie de des-cubrimiento de la propia impotencia frente al absolutismo de la realidad y de la necesidad de la onomástica y la narración, precisamente, como tácticas de distanciamiento (p. 61).
\end{abstract}

En la actualidad, las producciones provenientes de los medios de comunicación, como los programas de televisión, satisfacen las necesidades que surgen del enfrentamiento del absolutismo de la realidad, contribuyendo a la construcción de la estructura mítica de la sociedad, o a lo que Barthes "llama mito y que constituye, en esencia, un modo de comprender la forma en la que se estructuran los juegos de lenguaje en un entramado social" (Uribe-Jongbloed y Roncallo-Dow, 2013, p. 180). Así pues, estas narrativas buscan suscitar respuestas emocionales: asombro, maravilla, un sentido de propósito o sentido personal, alegría y participación, que, en esencia, son lo que llevaría a Roncallo-Dow (2008) a explicar, citando a Blumenberg,

Así, poesía o terror, y he aquí el componente dialéctico del problema, se presentan como modos de mantener distancia del absolutismo de la realidad, pues, "indudablemente, uno de los métodos elementales y acreditados de afrontar la oscuridad consiste no sólo en temblar, sino también en cantar" (p. 63). ${ }^{4}$

4 Segmento de Blumenberg citado por el autor tomado de Blumenber (2003). 
Dentro de estas respuestas emocionales, el asombro se manifiesta en una mezcla de admiración y miedo, una cierta reverencia hacia cosas que se perciben inmensas, poderosas, trascendentes y misteriosas. La maravilla, por su parte, es la capacidad de asombrarse ante los componentes extraordinarios del mundo del mito. Asimismo, estos textos suscitan cierta alegría, que surge del disfrute al contemplar la virtud representada o la belleza. Por sus parte, el sentido de propósito es clave en el distanciamiento del absolutismo de la realidad, puesto que comunica la idea de que la vida tiene sentido y de que todo tiene su propósito. Finalmente, otra de las reacciones decisivas del mitotipo es la participación, un sentido de pertenencia a algo importante, una cierta noción de que el mundo necesita de nuestra ayuda, que podemos hacer la diferencia.

En definitiva, el mitotipo provee una estructura al texto que le facilita una conexión con la audiencia de carácter emocional, lo cual amplía su alcance, pese a los estilos emocionales de las diversas culturas. Por otra parte, cabe destacar que en culturas particulares el mitotipo representa al mito, ya que en él se encuentra una serie de particularidades, arquetipos y unos códigos fácilmente reconocibles por ese grupo de personas, lo que hace que sea un fuerte elemento a la hora de elaborar un contenido con vocación global.

\section{La morfología del mitotipo, modelo de análisis de contenidos transparentes}

En su trabajo, Olson se acerca a los contenidos transparentes para identificar y explicar en qué consisten algunas de sus estrategias narrativas y de producción para conseguir respuestas emocionales propias del mito. De este modo, ofrece un modelo análitico que facilita el reconocimiento de los elementos que contribuyen a una mayor compartibilidad de estos textos y que resultan idóneos para el logro de los objetivos del presente estudio. Por tanto, conviene exponer cada una de esas tácticas, ya que ellas constituyen los elementos por determinar en la experiencia de Year Zero.

En su análisis de los textos que emplean el mitotipo, Olson identifica diez atributos canalizadores de transparencia. Ocho corresponden al diseño narrativo: la neguentropía, la circularidad, la apertura, la virtualidad, 
la elipticalidad, el uso de arquetipos, la inclusión y la verosimilitud. Los otros dos restantes se refieren a los valores de producción y a la omnipresencia. Los primeros llaman la atención sobre la calidad de la ejecución del contenido, y causan asombro y admiración. La segunda promueve la interacción del público con estos contenidos.

La neguentropía, término usado por Kubey y Csikszentmihalyi (1990), describe la fuerza psicológica que produce satisfacción en la audiencia ante la coherencia y la familiaridad hallada en un texto. Esta reacción que producen los contenidos audiovisuales se basa en la familiaridad que tienen los espectadores con la fórmula que se emplea en la ficción y que se produce a partir de la repetición de esa receta. Una pauta que genera ciertas expectativas en el público respecto de rasgos presentes en el texto, como el género o el uso de arquetipos, y que constituye la construcción de un mundo ideal sin el desorden de la realidad.

Asimismo, muchos programas de televisión y películas poseen estructuras narrativas que describen la circularidad propia del monomito, en las que se produce un eterno retorno. Un regreso al hogar, que refuerza la idea en la gente de que todo tiene su propósito, ya que, pese a los peligros del viaje, este sirve para el perfeccionamiento propio y la felicidad al regresar al lugar del que se partió. En tal sentido, infunde esperanza en tiempos mejores tras la tribulación.

Este tipo de estructura puede o no presentarse de manera conclusiva, cerrada, en historias que no tienen continuación. Sin embargo, muchos de los textos transparentes apelan a la apertura de una narrativa serial, que facilita la extensión y expansión del relato, y cuya popularidad se aprecia en series y secuelas. Esta apelación a las raíces profundas de la cultura se aplica también a la creación de personajes, mediante el empleo de arquetipos, figuras que se comprometen con la aventura, en la que experimentan el miedo, el amor, sacrifican todo por el bien común o no, que pueden infundir admiración y suscitar empatía, uno de los primeros rasgos que promueve la participación vicaria de la audiencia con lo que se le cuenta. Así es 
como consigue, en primera instancia, la inclusión del público en la narrativa, resolviendo su deseo de conocer y participar en lo que la trama cuenta.

En este sentido, la elipticalidad, entendida como el aparato narrativo, mediante el cual se construye el espacio y, en especial, el tiempo: el orden, la frecuencia, la duración, el humor y la voz, constituye otras de las herramientas para reclamar la participación de la audiencia, la cual debe hacer inferencias con los datos que la trama le va proveyendo. Además, estos textos siguen un modo de representación institucional, en el que prima la verosimilitud, su narrativa emplea signos icónicos, sus significantes parecen tener una asociación natural con el significado y su apariencia es de inmediatez y de literalidad. Algo que también consigue la virtualidad, que es la sensación de realidad producida por la técnica de realización.

Nada de lo anterior sería lo suficientemente eficaz sin atender a aspectos externos al diseño narrativo, en su sentido estricto. Es decir, aquellos que conciernen a la realización y su introducción en el mercado. Así, en la morfología del mitotipo, los valores de producción, entendidos como los aspectos técnicos que intervienen en la realización del contenido, tienen un peso específico, por lo que deben mostrar calidad y pulimiento. No es preciso que sean épicos, pero sí logrados y efectivos y contribuyan al disfrute y la conexión con el texto. Por su parte, el modo en que se introduce el contenido transparente en la sociedad debe gozar de cierta omnipresencia con miras a facilitar una familiaridad entre el producto y el público, mediante el acceso a su conocimiento. De este modo, ambos aspectos canalizadores de transparencia están llamados a despertar emociones fundamentales para el mitotipo: el primero llama la atención sobre la calidad de la ejecución del contenido, y causa asombro y admiración. El segundo, promueve la interacción del público con estos contenidos.

Teniendo como base lo anterior y basándonos en la investigación en fuentes documentales sobre la experiencia de Year Zero, analizaremos las estrategias empleadas en la búsqueda de elementos de carácter mitotípicos y explorar el modo en que estas actuaron en el público internacional facilitando su compartibilidad. 


\section{Year Zero, trascendiendo las fronteras del álbum}

Year Zero es una "experiencia musical” (Wright, 2011, p. 97), basada en un universo narrativo complejo expresado a través de un entramado de productos compuestos por un disco, titulado con el mismo nombre, y un juego de realidad alternativa, con el que se crea una vivencia de inmersión, interactiva, usando un amplio rango de canales y tecnología de distribución (Wolf, 2012, pp.48-51). Normalmente, este tipo de juegos ofrece un relato fragmentado que debe ser resuelto a través de la colaboración y el esfuerzo entre los participantes (Phillips 2005, citado en Janes, 2013, p. 59).

Year Zero presenta un mundo distópico en los Estados Unidos de 2022 o año del renacimiento de América. Un futuro desde el que un grupo de disidentes envía a los ciudadanos del mundo, en 2007, una serie de claves informativas con el fin de que cambien el curso de los acontecimientos y eviten así la situación que padecen en su tiempo posapocalíptico: luego de varios ataques terroristas en Los Ángeles y Seattle, el Estado teocrático que dirige al país se arroga el control de la población en todos los aspectos de su vida, mediante la acción de entes gubernamentales y religiosos, así como por el vertido de una droga en los acueductos públicos.

La distopía, como base del mundo narrativo de Year Zero, es una de las elecciones clave de su exitosa inserción en varios mercados culturales. Este género no solo constituye un canal idóneo para las reivindicaciones que inspiraron a Reznor las letras del álbum, sino que también proporciona un cimiento firme al universo del relato, en el que se puede intuir un sentido y un orden, en la forma que el mito lo ofrece. Su estructura y los elementos propios del género crean una fuerte neguentropía.

En tal sentido, el capital cultural empleado en la creación del universo de Year Zero hace claras referencias a la literatura distópica clásica de comienzos del siglo pasado: 1984 (Orwell, 2013), Un mundo feliz (Huxley, 2013 ) y Fahrenheit 451 (Bradbury, 1993). Y, como es natural en este género, la ficción se enraíza en una realidad política y social que el conflicto central de la trama evoca. En este caso, se trata de la preocupación debida a la 
erosión de las libertades civiles en nombre de la seguridad nacional, a consecuencia de la USA Patriot Act. Inquietud que también se halla presente en buena parte de la producción audiovisual estadounidense de la primera década del siglo XXI (Mirrlees, 2005; Sánchez-Escalonilla, 2009).

A todo esto se suma el componente místico de la historia, representado en su propio título, en el que se alude al fin del mundo y, sobre todo, por la introducción de un misterioso ente, conocido como "la presencia". Este elemento clave de la trama hace referencia al avistamiento, por parte de algunos personajes, de un par de brazos extendidos hacia la tierra, desde el cielo. Al comienzo, los jugadores pensaron que se trataba de una alucinación producida por alguna droga. Luego, lo asociaron a Dios o a otra fuerza sobrenatural. Aún sin definirse, quedó claro que se trataba de un fenómeno físico que se podía registrar con una cámara o crear distorsión sonora, como se apreciaba al final de My Violent Heart, el tema contenido en el primer USB dejado en un baño del recinto del concierto de Nine Inch Nails, en Lisboa.

El impulso evocador del juego y su inserción en las tendencias narrativas del momento lo hicieron más eficaz en el mercado internacional. El halo de misterio y de misticismo que cubría las pistas llevó a los fanes a comentar en línea los datos y a relacionarlos con la Biblia o producciones, como $V$ de vendetta (Moore, 1989) e Hijos de los hombres (Cuarón, 2006). La experiencia de los participantes, al intentar darles sentido a las pistas ofrecidas por los storymaster, reforzó las reacciones emocionales asociadas al mito: la maravilla por las apariciones de la presencia, el asombro ante la magnitud de la aventura, la conciencia de los poderes en pugna, así como un sentido de propósito de la experiencia.

Este sentido de propósito se desplegó en especial en la organización del juego y la manera en que este recordó la estructura del género. La narración comenzaba in media res, cuando grupos disidentes del futuro hacen contacto con el pasado. El detonante de la acción estaba prescrito en las primeras acciones que los storymaster animaban al participante a tomar: encontrar las claves que los llevaran a la "madriguera de conejo", el si- 
tio $w w w$.iamtryingtobeleieve.com $y$, una vez ahí, navegar hacia otras webs del juego, compartir información o ponerse en contacto con el administrador vía correo electrónico.

El desarrollo de la acción transcurría en la solución de acertijos propuestos mediante teléfono o internet y su discusión en foros. Al llegar al punto álgido, en el año cero, relatado por algún personaje en el disco y en las webs, un avistamiento de la presencia sobre el capitolio sugiere el final del mundo. Entretanto, en 2007, algunos fanes de Los Ángeles fueron convocados a una reunión secreta, que terminó siendo un concierto de Nine Inch Nails. A mitad de este, un equipo SWAT (special weapons and tactics 'armas especiales y tácticas'), que hacía parte del juego, interrumpió el evento por tratarse de una reunión clandestina. Los presentes fueron animados a volver a sus casas en el transporte que los llevó hasta allí, con la promesa de que serían contactados nuevamente.

Así, el desenlace de esta experiencia combina la circularidad que suponen los relatos del regreso a casa, como el monomito, con la apertura propia de la narrativa serial al final de una temporada. En ellas, lo que da sentido al relato no se resuelve del todo, y deja la narrativa eternamente diferida (Hills, 2004, citado en Green, 2005). Es más, cada uno de los eventos programados para apoyar la gira mundial de Nine Inch Nails y la promoción del disco funcionaron a modo de episodios de una serie de televisión, donde cada acción generaba más y más información sobre el mundo de Year Zero, al tiempo que proponía una nueva intriga por resolver.

Es interesante notar cómo la elipticalidad de esta experiencia fue fundamental en su creación, pese a la frecuencia desigual con la que se introdujeron las pistas que guiaban la narrativa. El interés de los fanes no decreció, sino que generó una mayor expectativa, discusión y cooperación en línea, en foros creados en las webs preparadas ad hoc para comunicar y evocar el mundo alternativo. Desde la perspectiva antropológica, esta estrategia apeló a la pulsión escópica y originó la noción de estar participando en un sistema mítico común. 
Asimismo, las estrategias de los creativos de 42 Entertainment se dirigieron a potenciar estas sensaciones, afianzando el sentido de propósito, mediante la virtualidad y la verosimilitud de la experiencia que proponían a los fanes. Al crear una realidad alterna, monitorizaron las acciones de los participantes, atendiendo a su comportamiento impredecible, casi en tiempo real. De este modo, ofrecían al participante "una ilusión de control, que no era necesariamente el control en sí mismo”, como Janes señala que debe ocurrir en un ARG (2013, p. 67).

En una ocasión, algunas webs del proyecto incluyeron, con fines decorativos, fragmentos de Alguien voló sobre el nido del Cuco (Kesey, 1962) y Matadero cinco (Vonnegut, 1969). Sin embargo, los fanes entendieron esta acción como nuevas pistas, que comenzaron a discutir en foros. La respuesta de los storymaster no se hizo esperar, e introdujeron estos títulos dentro de la historia de Year Zero como textos prohibidos por el régimen (Rose, 2007). En definitiva, esta interacción entre la libertad de participar y sentirse seguro en los límites de la creación narrativa coincide con el deseo de orden y control que debe proveer el mitotipo, al tiempo que genera un universal producido por la empresa, a partir de un universal creado por la audiencia.

Por otro lado, esta simulación de control fue favorecida por el alto grado de virtualidad que supone un juego de inmersión como este, en el que, además de los medios digitales, se apela a situaciones que se desarrollan en la vida física, en las que las reacciones emocionales propias de la inclusión están presentes de una manera más fuerte. Cada elemento de la estrategia lleva al participante a experimentar en primera persona el desarrollo de la trama. La carga afectiva resultante del avance de la acción, no de manera vicaria sino de manera real, contribuyó también a un refuerzo de la neguentropía, mediante una conciencia de que lo que se vive es cierto, aunque sea fruto de un universo construido, en el que los participantes se mezclan con los personajes del juego.

La introducción de personajes y entidades (corporaciones y despachos) de la ficción, que sustenta este universo narrativo, se produce a través de los medios de interacción con los fanes: las canciones del disco, que 
relatan los conflictos que ellos viven, los sitios webs, donde también se presentan en videos y en apariciones personales. Es el caso de uno de los líderes de la resistencia, Neil Czerno, interpretado por Monti Sharp, quien pronuncia un discurso en el concierto final de Los Ángeles, para animar al auditorio a abrir los ojos y a luchar para cambiar el mundo (Charles y Hall, 2009). Así, villanos, mártires y héroes que conforman la historia interpelan al jugador, y lo incluyen en el mundo del relato y lo hacen parte indispensable de la resolución de la trama.

En definitiva, el carácter épico de la narración se convierte en un elemento más de transparencia, en dos sentidos: en la evocación de arquetipos y en el de la inclusión. Los primeros originan reconocimiento y admiración, disfrute ante la acción valerosa del personaje, que supone la ejecución del conflicto básico. Y el segundo atributo anima a la participación, reforzando la comunidad de los fanes, que forman parte de un ritual común, donde el disfrute tiene una causa en el reconocimiento de valores y emociones. En este sentido, cabe notar que el componente de clandestinidad de las acciones del juego también suscita cierto placer por el misterio que supone la aventura.

Llegados a este punto, hay que decir con Olson que "no todas las manifestaciones afectivas del mitotipo son narratológicas" (Olson, 1999, p. 88). Los aspectos relativos a los valores de producción, que intervienen en la realización de contenidos, y la omnipresencia conseguida a partir de universales creados por la compañía, y que se verían reforzados por los universales creados por la audiencia, mediante las distintas vías de distribución, son elementos decisivos para lograr la transparencia de un producto $y$, por tanto, aumentar su grado de compartibilidad en el mercado internacional.

En el caso que nos ocupa, la calidad en la ejecución del contenido y la forma en que se introdujo al público fue el fruto de una feliz reunión: la de un artista muy particular, Trent Reznor, con una exitosa empresa de entretenimiento inmersivo, 42 Entertainment, famosa por campañas como Why So Serius?, para la promoción de The Dark Knight (Nolan, 2008), 
para Warner Bros, entre otras experiencias, anteriores y posteriores al disco. ${ }^{5}$ Sin la creatividad de ambos, no se hubiera podido comunicar de una manera tan clara el mundo de Year Zero.

Si antes señalamos la importancia de la elección de la distopía como un género idóneo para comunicar las reivindicaciones de Reznor, la estrategia de desarrollar un ARG las llevó al plano de lo concreto. En este sentido, los valores de producción desempeñaron un papel decisivo para apuntalar la verosimilitud, la virtualidad, la inclusión y la neguentropía que se había construido con los elementos narrativos de la experiencia. Asimismo, cabe resaltar la comprensión, por parte de los productores, del tipo de dinámicas con las que su audiencia estaba relativamente familiarizada como parte del capital cultural, que, bien manejado, acorta las posibles brechas que pudieran surgir de las lagunas de producción o de capital.

42 Entertainment dispuso de treinta webs para que los fanes pudieran seguir y deducir la respuesta de las pistas dejadas en el merchandising: camisetas, flyers, dispositivos de USB y murales. Asimismo, tuvieron tres números de teléfono activos desde los que se daban nuevos indicios e instrucciones. Además de las acciones en los conciertos, hay que mencionar la capa de cromado térmico del CD de Year Zero. Al reproducirse, cambiaba de color y desvelaba la dirección de un sitio en internet y una advertencia de la Oficina de Moralidad, en la que pone bajo sospecha a quienes tuvieran el disco en sus manos, por escuchar material prohibido. De este modo, la omnipresencia de los elementos del juego, la calidad en los dispositivos mediante los cuales se llevó a cabo la experiencia y la inteligencia colectiva que generaba la colaboración entre los participantes fueron cruciales en su transparencia.

Por otra parte, como se dijo en la introducción, la realidad enriquecía el contenido del disco y del juego. Tras los atentados del 11 de septiem-

542 Entertainment es una de las agencias de productos transmedia y juegos de realidad alternativa más reconocidas en el medio. Entre sus producciones, se encuentran algunas de las experiencias más emblemáticas de la producción transmedia, como Why So Serious? y I Love Bees. Además de esto, de las filas de 42 Entertainment se han desprendido múltiples compañías formadas por algunos de sus excreativos, como No Mimes Media, Fourthwall Studios y Smith and Tinker. 
bre de 2001, la Cumbre de las Azores, en 2003, en la que se decide dar un ultimátum al régimen de Sadam Husein, y los atentados de Londres 2005 y Madrid 2004, surgió una seria preocupación por el curso que estaba tomando el comienzo del nuevo siglo sobre política exterior y seguridad internacional. Por tanto, en relación con estos temas, había una proximidad cultural de la audiencia estadounidense y europea que vivió el ARG, respecto del mundo distópico del disco y la lucha que este promovía. No en vano, varias de las acciones del juego se dieron en países implicados en la guerra de Irak, como Portugal y España, en especial críticos con el tema. Este elemento de creación del universo de la historia puede ser entendido desde las nociones de mundo sobrepuesto (overlaid world) presentada por Wolf (2012, p. 28), y que está elaborada sobre la construcción del mundo primario y secundario de Tolkien (Tolkien, 1947; Wolf, 2012). En este sentido, Wolf destaca el uso de los elementos que la audiencia conoce por defecto del mundo primario como un potencial fortalecedor de la sensación de Gestalten, o de forma completa, del mundo subcreado (Wolf, 2012, p. 53).

A esto se sumaron las continuas desavenencias entre Reznor y Universal Music Group, dueña de su discográfica, Interscope Record, respecto de la distribución y el precio del CD. En este último tema, la empresa argumentaba la necesidad de incrementar el valor del disco, debido a las mejoras que se le habían incorporado para que revelara las pistas del juego al reproducirse. Una diferencia que el líder de la banda había cubierto con su propio dinero y, por tanto, no estaba de acuerdo con que se le cobrara a sus seguidores.

Por eso, mediante las acciones propuestas por los storymaster, los jugadores pudieron estrenar, distribuir y modificar algunos temas incluidos en el álbum, gracias a las canciones que se incluyeron como indicios en dispositivos USB. Como era previsible, Year Zero fue la última producción de Nine Inch Nails con esta discográfica.

En definitiva, la actitud de Reznor dotó de honestidad a la propuesta del álbum y enriqueció la experiencia diseñada por 42 Entertainment. Los fanes de Nine Inch Nails podían sentir que el líder de la banda era par- 
te real y sincera del sistema mitotípico común y, en ese sentido, evocaba al héroe, que los incluía en una lucha en la que eran defendidos de la poderosa fuerza de la industria musical. La lógica detrás de la creación de esta experiencia tanto en su factura como en la implementación de la campaña suscitó una serie de reacciones relacionadas con el mito: asombro, disfrute, maravilla y sentido de propósito.

\section{Conclusiones}

Si vemos a Year Zero como un ejemplo de buen empleo de estrategias narratológicas y de producción orientadas a la proyección internacional de un contenido, basadas en los anhelos más profundos del hombre, a partir del mitotipo, logramos darnos cuenta de lo lejos que pueden llegar dichas estrategias por fuera de las formas de producción tradicional. En muchos sentidos, la transparencia y la compartibilidad cultural han sido vistas como estrategias basadas en las características de una narrativa contenida en una sola pieza textual que se relacionan o no con un mercado particular; sin embargo, el caso analizado nos muestra cómo se pueden atenuar posibles lagunas culturales a través de la utilización de múltiples piezas textuales que apelan a diferentes sensibilidades, que hacen ubicuo el contenido y que, por ende, lo hacen relevante en un contexto, y que, además, generan una red de significados que educan y a la vez absorben el capital cultural de la audiencia para que esta pueda decodificar el texto y participar de él.

Los aspectos canalizadores de transparencia de Year Zero destacan por la originalidad con la que presentaron una narrativa de hondas raíces clásicas, mediante nuevas tecnologías y, en especial, en un juego de inmersión a gran escala, diseñado de manera extraordinaria, para reivindicar temáticas de actualidad en el momento en que se produjo la experiencia y que también estaban presentes en el tratamiento de otras producciones exitosas globales.

Los temas que Rezno trató en la experiencia no se sentían locales sino globales. Vivimos en un mundo cada vez más conectado y complejo, al que apenas alcanzamos a comprender. Pese a los avances tecnológicos, la política, el destino del hombre parece tomar un rumbo indeseable en diver- 
sos temas. En este sentido, la historia de Year Zero apunta al íntimo deseo de convertirnos en los dueños de nuestro destino y cambiar el rumbo y lo ata a las inquietudes que se reflejan en parte de la construcción mítica de nuestros tiempos, nuestro Zeitgeist.

Sin duda, al apelar al componente antropológico mediante estrategias mitotípicas, ayudó a componer un contenido aún más global, reconocible en todos sus componentes, accesible, que logró la implicación de la audiencia, la mejor prueba de su efectividad.

\section{Referencias}

Allen, G. (2011). Intertextuality (2. ${ }^{\mathrm{a}}$ ed.). Londres y Nueva York: Routledge.

Bartrina, F. (2004). The challenge of research in audiovisual translation. En P. Orero (ed.), Topics in Audiovisual Translation (pp. 157-167). Ámsterdam y Philadelphia: John Benjamins Publishing Company.

Blumenberg, H. (1985). Work on Myth. Cambridge: MIT Press.

Blumenberg, H. (2003). Trabajo sobre el mito. Barcelona: Paidós.

Bourdieu, P. (1984). Distinction: A Social Critique of the Judgment of Taste. Cambridge, MA: Harvard University Press.

Bradbury, R. (1993). Fahrenheit 451. Almería: Ediciones perdidas.

Brown, S. C. (2011). Artist Autonomy in a Digital Era: The Case of Nine Inch Nails. Empirical Musicology Review, 6(4), 198-213. Recuperado de http:/ / ezproxy.lib.ed.ac.uk/login?url=http:// search.ebscohost.com/login.aspx ?direct $=$ trueydb $=$ afhyAN $=83259125$ ysite $=$ eds-liveyscope $=$ cite

Cattrysse, P. (1992). Film (Adaptation) as Translation: Some Methodological Proposals. TARGET: International Journal of Translation Studies, 4(1), 53-70. 
Charles, A. y Hall, O. (2009). "I am Trying to Believe": Dystopia as Utopia in the Year Zero Alternate Reality Game. Eludamos. Journal for Computer Game Culture, 3(1), 69-82. Recuperado de http:// www.eludamos.org/index.php/eludamos/article/viewArticle/ vol3no1-8/111

Chiaro, D. (2008). Issues in Audiovisual Translation. En J. Munday (ed.), The Routledge Companion to Translation Studies (pp. 141-165). Oxon y Nueva York: Routledge.

Conway, K. (2012). Cultural Translation. En Handbook of Translation Studies (vol. 3). John Benjamins.

Cuarón, A. (2006). Children of Men. USA: Universal Pictures.

Davis, C. H. y Nadler, J. (2010). International Television Co-productions and the Cultural Discount: The Case of Family Biz, a Comedy. En 9th World Media Management and Economics Conference. Bogotá. Recuperado de http://www.ryerson.ca/ c5davis/publications/Nadler - Davis - International Television Coproduction v7 - 12 May 2010.pdf

Fung, A. (2004). Coping, cloning and copying. En A. Moran y M. Keane (eds.), Television Across Asia: Television Industries, Programme Formats And Globalization. Londres y Nueva York: Routledge.

Gray, J. (2010). Show Sold Separately: Promos, Spoilers, and Other Media Paratexts. Nueva York y Londres: Nueva York University Press.

Green, J. (2005). People, American Teen Dramas, and Australian Television 1992-2004. Queenaland University of Technology.

Hogg, C. (2013). Cracking the USA? Interpreting UK-to-US TV drama translations. New Review of Film and Television Studies, 11(2), 111 132. doi.org/10.1080/17400309.2012.708266 
Hoskins, C. y Mirus, R. (1988). Reasons for the US Dominance of the International Trade in Television Programmes. Media, Culture y Society, 10(4), 499-504. doi.org/10.1177/016344388010004006

Huxley, A. (2013). Un mundo feliz. México: Porrúa.

Iwabuchi, K. (2010). Taking “Japanization” Seriously: Cultural Globalization Reconsidered. En D. K. Thussu (ed.), International Communication: A Reader (pp. 410-433). Oxon y Nueva York: Routledge.

Janes, S. (2013). Players and Puppetmasters: Alternate Reality Game and Negotiated Consumer/Producer Relationship. Networking Knowledge, 6(4), 59-73.

Jenkins, H. (2006a). Convergence Culture: Where Old and New Media Collide. Nueva York y Londres: Nueva York University Press.

Jenkins, H. (2006b). Searching for the Origamy Unicorn: The Matrix and Transmedia Storytelling. En Convergence Culture: Where Old and New Media Collide. Nueva York y Londres: Nueva York University Press.

Jenkins, H., Ford, S. y Green, J. (2013). Spreadable Media: Creating Value and Meaning in a Network Culture. Nueva York y Londres: Nueva York University Press.

Kesey, K. (1962). One Flew Over the Cuckoo's Nest. Estados Unidos: Viking Press.

Kubey, R. y Csikszentmihalyi, M. (1990). Television and the Quality of Life: How Viewing Shapes Everyday Experience. Hillsdale, NJ: Lawrence Eribaum Associates.

Lee, F. L. F. (2006). Cultural Discount and Cross-Culture Predictability: Examining the Box Office Performance of American Movies in Hong Kong. Journal of Media Economics, 19(4), 259-278. doi. org/10.1207/s15327736me1904_3 
Maslow, A. (1987). Motivation and Personality. Nueva York: Harper and Row.

Mirrlees, T. (2005). Hollywood's Uncritical Dystopias. Cineaction: Canada's Leading Film Studies Journal, 95(1), 4-15.

Moore, A. (1989). V for Vendetta. Vértigo.

Moran, A. (ed.) (2009). TV Formats Worldwide: Localizing Global Programs. Bristol and Chicago: Intellect.

Nolan, C. (2008). The Dark Knight. USA: Warner Bros. Pictures.

Olson, S. R. (1999). Hollywood Planet: Global Media and the Compettitive Advantage of Narrative Transparency. Mahwah, NJ y Londres: Lawrence Erlbaum Associates, Inc.

Orwell, G. (2013). 1984. México: Lectorum.

Place, J. A. (1974). The Western of John Ford. Seacaucus: Citadel Press.

Reznor, T. (2007). Year Zero. USA: 42entertainment.

Rivera-Betancur, J. L. y Uribe-Jongbloed, E. (2012). La suerte de la fea muchas la desean: de "Yo soy Betty, la fea" a "Ugly Betty". En M. Pérez (ed.), Previously On (pp. 825-842). Salamanca: Universidad de Salamanca.

Rohn, U. (2011). acuna or Universal? Introducing a New Model for Understanding Crosscultural Audience Demand. Media Culture Society, 33(4), 631-641. doi.org/10.1177/0163443711399223

Roncallo-Dow, S. (2008). El juego de la distancia: entre la significatividad yla recepción: un viaje por los prometeos de Blumenberg. Universitas Philosophica, 25(51), 59-83. 
Rose, F. (2007). Secret Website, Coded Messages: The New Worl Inmersive Game. Wire Magazine. Recuperado de http://archive.wired. com/entertainment/music/magazine/16-01/ff_args\#end

Sakellariou, P. (2014). The Appropriation of the Concept of Intertextuality for Translation-Theoretic Purposes. Translation Studies, 8(1), 35-47. doi.org/10.1080/14781700.2014.943677

Sánchez-Escalonilla, A. (2009). Hollywood y el arquetipo del atrincherado: clave dramática y discurso político del 11-S. Revista Latina de Comunicación Social, 64, 926-937. doi.org/10.4185/RLCS-642009-871-926-937

Singhal, A. y Svenkerud, P.J. (1994). Pro-socially Shareable Entertainment Television Programmes: A Programming Alternative in Developing Countries? The Journal of Development Communication, 5(2), 17-30.

Singhal, A. y Udornpim, K. (1997). Cultural Shareability, Archetypes and Television Soaps. Gazette, 59(3), 171-188.

Steinberg, M. (2012). Anime's Media Mix: Franchising Toys and Characters in Japan. Minneapolis, Londres: University of Minnesota Press.

Straubhaar, J. D. (1991). Beyond Media Imperialism: Asymmetrical Interdependence and Cultural Proximity. Critical Studies in Mass Comunication, 8(1), 39-59.

Straubhaar, J. D. (2007). World Television: From Global to Local. Londres, Los Ángeles, Nueva Delhi y Singapur: Sage Publications.

Thussu, D. K. (2010). Mapping Global Media Flow and Contra-Flow. En D. K. Thussu (ed.), International Communication: A Reader (pp. 221-238). Oxon y Nueva York: Routledge.

Tolkien, J. R. R. (1947). On Fairy Stories. En Essays Presented to Charles Williams. Oxford y Londres: Oxford University Press. 
Uribe-Jongbloed, E. y Espinosa-Medina, H. D. (2014). A Clearer Picture: Towards a New Framework for The Study of Cultural Transduction in Audiovisual Market Trades. OBSERVATORIO (OBS*), 8(1), 23-48. Recuperado de http://obs.obercom.pt/index.php/ obs/article/view/707/642

Uribe-Jongbloed, E. y Roncallo-Dow, S. (2013). El medio es el mito: entre McLuhan y Barthes. Universitas Philosophica, 30(61), 177-203. Recuperado de http://revistas.javeriana.edu.co/index.php/vniphilosophica/article/view/10642

Vonnegut, K. (1969). Slaughterhouse-Five, or The Children's Crusade: A Duty-Dance with Death. Delacorte.

Wang, G. y Yeh, E. Y. (2005). Globalization and Hybridization in Cultural Products: The Cases of Mulan and Crouching Tiger, Hidden Dragon. International Journal of Cultural Studies, 8(2), 175-193. doi. org/10.1177/1367877905052416

Wolf, M. J. P. (2012). Building Imaginary Worlds: The Theory and History of Subcreation. Nueva York y Londres: Routledge.

Wright, K. (2011). Nine Inch Nails' Year Zero and the Biopolitics of Media Convergence. En B. Flota y J. P. Fisher (eds.), The Politics of Post9/11 Music: Sound, Trauma, and the Music Industry in the Time of Terror. Farnham and Burlington: Ashgate Publishing, Ltd. 